\title{
Solving Time-Fractional Diffusion Equation: A Finite-Element Approach
}

\author{
Hussein J. Zekri ${ }^{\text {a }}$ \\ ${ }^{a}$ Mechanical Engineering Department, University of Zakho, Kurdistan Region, Iraq - (Hussein.zekri@uoz.edu.krd)
}

\begin{abstract}
:
The numerical solution for a time-fractional diffusion equation supplemented with initial and boundary conditions is considered. The scheme is based on the Galerkin finite element method. The uniform space discretization is applied to study the stability of the solution of the problem within our approach. An analytically solvable example is presented to make a comparison between the exact solution and our numerical solution. By presenting the absolute error with different step-sizes and different values for timefractional derivative, reliability and efficiency of our proposed numerical method is manifested.
\end{abstract}

KEYWORDS: Time-Fractional Diffusion Equation, Finite Element Method.

\section{INTRODUCTION}

Modelling real life phenomena are, in many cases, lead to Initial-Boundary Value Problems (IBVPs) which highly depend on the initial and boundary behaviour of the phenomena. Analytically solving these kind of problems (linear or non-linear) have always being a great challenge for researchers. However, when the integer order (time, space or both) of these problems is replaced by a fraction then the challenge is increased even further. This is simply because the analytical tools for fractional-order integration or differentiation is much more limited and restricted to some special cases or functions compared to integer order. Therefore, recently, seeking the numerical methods to solve fractional-order problems has attracted the attention of many researches. The fractional-order problems arise in modelling of many applications in different applied fields such as engineering, physics and some others. We do not mention any of these applications or any physical interpretation of a fractional derivative here, but for interested reader, they can be found in (Samko et al., 1993), (Hilfer, 2000), (Mainardi and Pagnini, 2003), (Kilbas et al., 2006), (Podlubny et al., 2009) and the references there in.

This paper focus on the numerical solution of 1D TimeFractional Diffusion Equation (TFDE) supplemented with initial and boundary conditions. This happens when the firstorder time derivative in the diffusion equation is replaced by a fraction, $\$ \backslash$ alpha $\$$ say, where $\$ 0<$ alpha $<1 \$$. In some modellings both orders of derivative (in time and space) are replaced with fractions. In this case it is called Time-Space Fractional Diffusion Equations (STFDEs). Different numerical approaches have been studied on STFDEs and TFDEs. For example, (Ling and Yamamoto, 2013) used the Jacobi Spectral-Collocation Method and applied the eigenfunction approach to find a highly accurate numerical solution for STFDE with initial and boundary conditions. A comparison was made by (Atangana, 2015) for the two wellknown methods, Crank-Nicholson and the forward scheme, on 3D TFDE and he found a more efficient numerical result via stability analysis using the former method. Also TFDE studied with different finite element approaches and schemes by (Esen et al., 2013), (Tasbozan et al., 2013), (Esen et al., 2014), and (Esen et al., 2015). They presented a comprehensive work when zero source function is considered in their studies. The Mittag-Leffler function is used as the basis functions in (Hanert, 2010) and (Hanret \& Piret 2012) for solving numerically the space-time fractional diffusion.

In this work, we consider the following 1D fractional diffusion equation

$$
\frac{\partial^{\propto} u}{\partial t^{\propto}}=k \frac{\partial^{2} u}{\partial x^{2}}+f
$$

where $0<\alpha<1, k$ is the dimensionless diffusivity constant which will be considered unity in this work and $f=f(x, t)$ is any given continuous functions in the domain $D=\{x: x \in$ $[0,1], t \geq 0\}$. It is worth mentioning that for $1<\alpha<2$, equation (1) represents the fractional diffusion-wave equation, see for example (Sun \& Wu, 2006). Also for $0<\alpha<2$, (Mainardi, 1996) used the Laplace transform to express the fundamental solution in terms of an auxiliary function for Cauchy and Signalling problems.

The fractional diffusion equation (1) is subject to the initial condition

$$
u(x, 0)=a(x)
$$

for all $x \in[0,1]$ and the boundary conditions

and

$$
u(0, t)=b(t)
$$

$$
u(1, t)=c(t)
$$

for all $t \geq 0$. The functions $a(x), b(t)$ and $c(t)$ are given continuous functions. The term $\partial^{\alpha} u / \partial t^{\alpha}$ on the left-hand side of equation (1) refers to the Caputo fractional-derivative of order $0<\alpha<1$ for the diffusion function $u=u(x, t)$, see (Samko et. al., 1993) and (Podlubny, 2009), which is defined as

$$
\frac{\partial^{\alpha} u}{\partial t^{\alpha}}=\frac{1}{\Gamma(1-\alpha)} \int_{0}^{t}(t-\tau)^{\alpha-1} \frac{\partial u}{\partial t} \mathrm{~d} \tau
$$

Where $\Gamma(\cdot)$ is the spacial Euler's Gamma function which is a generalization of the factorial function for non-integer numbers. There are more definitions that can be used instead of Caputo's for the fractional derivative and integral; the Riemann-Liouville 
definition, Erdelyi-Kober fractional integral and derivative, Hadamard part fractional integral and derivative, Riesz fractional integral and derivative, and Grunwald-Letnikov fractional derivative. They are related to each other. For the relation between the two definitions of fractional derivative and integral given by Caputo and Riemann-Liouville and their difference, see (Samko et. al., 1993) and (kilbas, 2006).

\section{WEAK FORMULATION}

Our approach in this section is to write the TFDE (1) in its weak form. Hence multiplying the TFDE (1) by the weight function $\Psi(x)$ and integrate both side over the interval $[0,1]$, that is

$$
\int_{0}^{1}\left(\frac{\partial^{\alpha} u}{\partial t^{\alpha}}-\frac{\partial^{2} u}{\partial x^{2}}\right) \Psi \mathrm{d} x=\int_{0}^{1} f \Psi \mathrm{d} x .
$$

For simplicity, we choose the boundary conditions (3-4) to be homogeneous. Hence, integration by parts for the second integral on the left-hand side of (6) leads to

$$
\int_{0}^{1} \frac{\partial^{\alpha} u}{\partial t^{\alpha}} \Psi \mathrm{d} x+\int_{0}^{1} \frac{\partial u}{\partial x} \frac{\mathrm{d} \Psi}{\mathrm{dx}} \mathrm{d} x=\int_{0}^{1} f \Psi \mathrm{d} x .
$$

The reason we rewrite the weak form (6) as (7) is to allow us to use the linear B-spline basis functions for representation of the function $u(x, t)$ which will be given in details in the next section. Now, we are at a place to let the piece-wise linear functions to represent the diffusion function $u(x, t)$ using the weak form (7).

\section{NUMERICAL SOLUTION}

In this section we follow the Galerkin finite element method to solve the TFDE problem (1-4). First the time and space will be discretized uniformly as follows. The time is divided into $N$ equally-spaced time steps (with length $\delta_{t}$ ), $t_{1}=$ $0, t_{2}, \ldots, t_{N-1}, t_{N}=T$, where the instants $t=0$ and $t=T$ are the initial and terminal time-steps, respectively. The space is partitioned into $M-1$ equal subintervals, $\left[x_{i}, x_{i+1}\right]$ for $i=1,2, \ldots, M-1$, with $\delta_{x}=x_{i+1}-x_{i}$, where $x_{1}=0$ and $x_{M}=1$.

With both the time and space discretized, we assume that the solution of the problem $(1-4), u(x, t)$, takes its approximation form in terms of piece-wise linear polynomial functions as follows

$$
u(x, t)=\sum_{i=0}^{M} \sum_{j=0}^{N} \Psi_{\mathrm{i}}(x) u_{i j} \varphi_{j}(t) .
$$

Here $u_{i j}$ are unknown coefficients which have to be determined and the set $\left\{\psi_{i}(x)\right\}_{i=1}^{i=M}$ consists of basis functions (linear B-spline) on the space domain and are defined as

$$
\psi_{i}(x)= \begin{cases}0 & ; x \leq x_{i-1} \\ x-x_{i} & ; x_{i-1} \leq x \leq x_{i} \\ x_{i+1}-x & ; x_{i} \leq x \leq x_{i+1} \\ 0 & ; x \leq x_{i-1}\end{cases}
$$

Where the Caputo's definition (5) of the basis functions given in (9) is

$$
\psi_{i}^{\alpha}(x)=\frac{\delta_{x}^{-1}}{\Gamma(2-\alpha)} \begin{cases}0 & ; x \leq x_{i-1} \\ x^{1-\alpha} & ; x_{i-1} \leq x \leq x_{i} \\ -x^{1-\alpha} & ; x_{i} \leq x \leq x_{i+1} \\ 0 & ; x \leq x_{i-1}\end{cases}
$$

It is shown in (Lin et al., 2020) that when Galerkin finite element method is followed the locality of the fractional derivative for the basis functions (9) is preserved. The set of the basis functions for time $\left\{\varphi_{j}(t)\right\}_{j=1}^{j=N}$ is to be defined with the same manner as $\left\{\psi_{i}(x)\right\}_{i=1}^{i=M}$ in (9).

Now, after time and space discretization and representing the solution $\$ \mathrm{u}(\mathrm{x}, \mathrm{t}) \$$ in terms of the basis functions we do the following. We substitute (8) into the weak form (7) and present the weight function $\Psi(x)$ by $\psi_{m}(x), 1 \leq m \leq M$, the piecewise linear functions (9). Also we multiply both sides of the resulted formulation with the linear B-spline for time, $\varphi_{n(t)}, 1 \leq$ $n \leq N$, and integrate it with respect to time over the time period $[0, T]$. These steps lead to a set of discrete equations which can be written into the following matrix form

$$
\Pi \Lambda \Upsilon^{\alpha}+\Omega \Lambda \Theta=\Sigma \text {. }
$$

Where for the time-derivative matrix $\Upsilon^{\alpha}$, the superscript $\alpha$ is used to easily distinguish the matrix that its terms deal with fractional derivative from the other matrices. The entries of the matrix $\Lambda$ are the unknowns coefficients $u_{i j}$ which will construct the solution $u(x, t)$ defined in (8). The matrices $\Pi, \Upsilon^{\alpha}, \Omega, \Theta$ and $\Sigma$ have the known entries $\Pi_{m i}, \Upsilon_{j n}^{\alpha}, \Omega_{m i}, \Theta_{j n} \$$ and $\Sigma_{m n}$, respectively, which are defined as follows

$$
\begin{gathered}
\Pi_{m i}=\int_{0}^{1} \psi_{m}(x) \psi_{i}(x) \mathrm{d} x \\
\Upsilon_{j n}^{\alpha}=\int_{0}^{T} \frac{\mathrm{d}^{\alpha} \varphi_{j}}{\mathrm{~d} t^{\alpha}}(t) \varphi_{n}(t) \mathrm{d} t \\
\Omega_{m i}=\int_{0}^{1} \frac{\mathrm{d} \psi_{m}}{\mathrm{~d} x}(x) \frac{\mathrm{d} \psi_{i}}{\mathrm{~d} x}(x) \mathrm{d} x \\
\Theta_{j n}=\int_{0}^{T} \varphi_{j}(t) \varphi_{n}(t) \mathrm{d} t \\
\Sigma_{m n}=\int_{0}^{1} \int_{0}^{T} \varphi_{m}(x) f(x, t) \varphi_{n}(t) \mathrm{d} t \mathrm{~d} x
\end{gathered}
$$

We recall that the integrand $f(x, t)$ in (16) of the source matrix $\Sigma$ is given. The entries, $\Pi_{m i}$ of the mass matrix $\Pi, \Omega_{m i}$ of the diffusion matrix $\Omega, \Theta_{j n}$ of the matrix $\Theta$ and $\Sigma_{m n}$ of the source matrix $\Sigma$, are integrated straightforward. However, in (13), the integrand term $\mathrm{d}^{\alpha} \varphi_{j} / \mathrm{d} t^{\alpha}$ with fractional-derivative will be differentiated using the Caputo's definition (5). Therefore, from (5) and (9-10), entries (13\}) can be rewritten as

$$
\Upsilon_{j n}^{\alpha}=\frac{\delta_{t}^{-1}}{\Gamma(2-\alpha)}\left(\int_{t_{j-1}}^{t_{j}}-\int_{t_{j}}^{t_{j+1}}\right) t^{1-\alpha} \varphi_{n}(t) \mathrm{d} t
$$

Where by Caputo's definition (5), the fractional derivative of a constant is evaluated to be zero. In (17), $\delta$ is the time-step.

It is clear that, the nature of these basis functions in finite element method is such that they always produce tri-diagonal matrices. All matrices, except $\Lambda$ and $\Sigma$, in system (11) are tri-diagonal. This will be helpful when we deal with very fine discretizations that lead to huge matrices and when their implementation cause memory efficiency problems with slow calculations' speed. To avoid this, for example in MATLAB, sparse matrix can be constructed.

To solve the system (11), we use the so-called Kronecker product, which allows us to rewrite the system (11) as the following

$$
\left(\Upsilon^{\alpha T} \otimes \Pi-\Omega^{\mathrm{T}} \otimes \Theta\right) \operatorname{vec}(\Lambda)=\operatorname{vec}(\Sigma) .
$$


In the new constructed system (18), the superscript " $T "$ stands for the transpose of the associated matrix, while the symbol " $\otimes$ " stands for the Kronecker product. For the definition of Kronecker product and its properties see (Bellman, 1997). Also in (18), vec( $\cdot)$ is a vector implemented by piling the columns of the matrix on top of one another. For the comparison of the current matrix approach and the matrix approach which is presented by (Podlubny et al., 2009), see (Hanert, 2010), section 4.

The existence of a unique solution of (18) is granted if and only if the matrices $\Pi, \Upsilon^{\alpha}, \Omega$, and $\Theta$ are non-singular, see (Horn \& Johnson, 1991). Therefore, we are required to test the singularity (the condition number of the matrix) of all matrices we have in (11) to ensure the existence and uniqueness of its solution. Otherwise, for ill-posed problem one should seeks, for example, the methods of regularization to improve the condition number. More details on regularizations can be found in (Tikhonov et al., 2013).

System (18) is to be supplemented with the given initial condition (2) and boundary conditions (3-4). Applying the same above conversation that led to (11), on the initial condition (2), it produces the following matrix equation

$$
\left(\Phi(0)^{T} \otimes \Pi\right) \operatorname{vec}(\Lambda)=\operatorname{vec}(A) .
$$

Where the entries of the matrix $A$ are

$$
A_{m}=\int_{0}^{1} \psi_{m}(x) a(x) \mathrm{d} x,
$$

for $1 \leq m \leq M$, and $\Phi(0)$ contains the elements of the sequence $\left\{\varphi_{j}(t)\right\}_{j=1}^{j=N}$ at the time instant $t=0$. Also the Galerkin formulation is to be applied on the two given boundary conditions (3-4). Therefore, the conditions (3) and (4) take the following matrix forms, respectively

and

$$
\begin{aligned}
& \left(\Theta^{T} \otimes \Psi(0)\right) \operatorname{vec}(\Lambda)=\operatorname{vec}(B), \\
& \left(\Theta^{T} \otimes \Psi(1)\right) \operatorname{vec}(\Lambda)=\operatorname{vec}(C) .
\end{aligned}
$$

Here $\Psi(0)$ and $\Psi(1)$ contain the elements of the sequence $\left\{\psi_{i}(x)\right\}_{i=1}^{i=M}$ at the endpoints of the space domain, $x=0$ and $x=1$, respectively, and the entries $B_{n}$ of $B$ and $C_{n}$ of $C$ are defined as

$$
B_{n}=\int_{0}^{1} \varphi_{n}(t) b(t) \mathrm{d} t
$$

and

$$
C_{n}=\int_{0}^{1} \varphi_{n}(t) c(t) \mathrm{d} t
$$

for $1 \leq n \leq N$.

The above-presented space and time discretization is uniform. The non-uniform mesh is usually applied to the unsteady problems with singularities which often occur in free-boundary problems, for example see (Ferziger \& Peric, 2012). In the next section, we demonstrate the above proposed scheme with an example. The space discretization is applied in two different number of grid points to examine the relative error of our numerical solution and the exact solution. This has been done to find the relative error response to the size of the subintervals $\left.\left[x_{i}, x_{i+1}\right\}\right], \delta_{x, i}=$ $x_{i+1}-x_{i}$ for $i=1,2, \ldots, M-1$, when the time-step held fixed. Although a finer grid mesh lead to more storage and require longer computational time, but attention has to be always taken to the solutions' accuracy and stability.

\section{NUMERICAL EXAMPLE}

In this section we analyse the numerical solution's performance of the problem (1-4) using the above proposed method by going through an example. The performance of our scheme will be demonstrated by comparing the numerical and analytical solutions. Although the presented scheme works for nonhomogeneous boundary conditions, for simplicity, homogeneous boundary conditions are chosen for the given example. Usually the analytical solution of such problems is beyond the capability of the present fractional analytical tools and cannot be found. That is why the numerical study of such problems is of great importance. However, for the reason of being able to compare our numerical solution with analytical solution, the timefractional initial-value problem $(25-27)$ is taken to be our illustrative example with known analytical solution.

We consider the governing equation to be the following timefractional diffusion equation

$$
\frac{\partial^{\propto} u}{\partial t^{\alpha}}=k \frac{\partial^{2} u}{\partial x^{2}}+\left(\frac{t^{1-\alpha}}{\Gamma(2-\alpha)}+\pi^{2}(t+1)\right) \sin (\pi t),
$$

defined in the domain $D=\{x: x \in[0,1], t \geq 0\}$. Equation (26) is supplemented with the initial condition

$$
u(x, 0)=\sin (\pi x), \quad 0 \leq x \leq 1,
$$

and the homogeneous boundary conditions

$$
u(0, t)=u(1, t)=0, t \geq 0
$$

The analytical solution of $(25-27)$ is $u(x, t)=(t+1) \sin (\pi x)$. Since non-uniform discretization is necessary for solution which has rapid changes or singularities and $u(x, t)=(t+1) \sin (\pi x)$ is smooth everywhere in the domain $D$, then we only perform uniform discretization. First, we implement the scheme with grid points $M=200$, space-step $\delta_{x}=0.005$, and time-step $\delta_{t}=$ $2.50 e-07$ with $\alpha=0.95$. The absolute error between both numerical and analytical solutions is shown in Figure 1a, which is of order $O\left(10^{-5}\right)$. Next, we halve the space-step with keeping the time-step unchanged, i.e. we divide the subintervals by two to validate the stability and convergence of our numerical solution. In Figure 1b, we found that as the grid points doubled (from $M=200$ with $\delta_{x}=0.005$ to $M=400$ with $\delta_{x}=$ $0.0025)$, the absolute error between both numerical and analytical solutions decreased by an order of $O\left(10^{-1}\right)$. Figures $1 \mathrm{a}-1 \mathrm{~b}$ show the decrease in absolute error between the analytical and numerical solutions when the grid points $M=200$ increased to $M=400$. The relative error has the same order of difference as the absolute error. As a result, increasing the mesh points leads to decreasing the absolute error and relative error, which confirms the convergence and stability of the present scheme. The same process has been carried out for $\alpha=0.9$ with keeping every other details fixed to monitor the absolute error while we decrease the value of $\alpha$. As it is shown in Table 1, there is an increase, roughly about an order of $O\left(10^{-1}\right)$. And as it is shown in Figures 2, as the fractional order $\alpha$ decreases, the absolute error increases. 


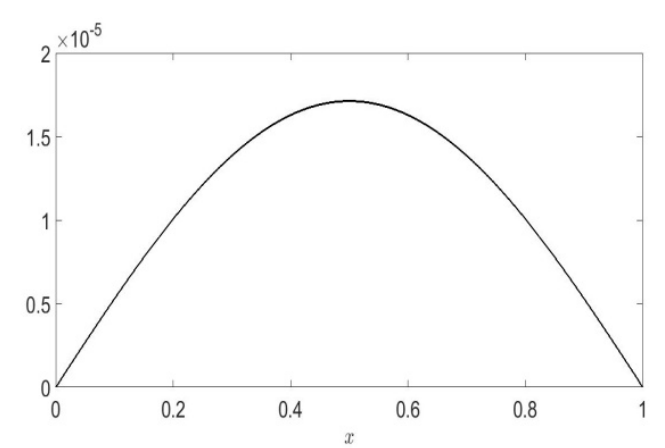

a) Number of grid points $M=200$. The absolute error is of order $O\left(10^{-5}\right)$.

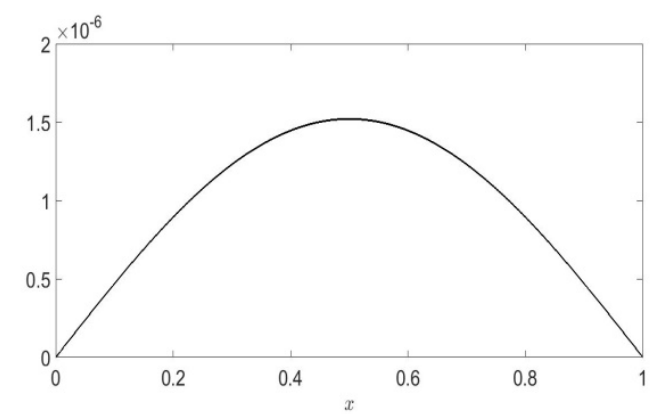

b) Number of grid points $M=400$. The absolute error is of order $O\left(10^{-6}\right)$.

Figure 1. The absolute error between our numerical solution and the analytical solution $u(x, t)=(t+1) \sin (\pi x)$ with $\delta=0.9$.

Table 1: Absolute error for linear B-spline with both stepsizes $\delta_{x}=0.005$ and $\delta_{x}=0.0025$ and time-step $\delta_{t}=2.50 \mathrm{e}-07$ for $\alpha=0.9$ and $\alpha=0.95$.

\begin{tabular}{crrrr}
\hline \multirow{2}{*}{$x$} & \multicolumn{2}{c}{$\alpha=0.9$} & \multicolumn{2}{c}{$\alpha=0.95$} \\
\cline { 2 - 5 } & $\delta_{x}=0.005$ & $\delta_{x}=0.0025$ & $\delta_{x}=0.005$ & $\delta_{x}=0.0025$ \\
\hline 0 & 0 & 0 & 0 & 0 \\
0.09 & $3.2079 \mathrm{e}-06$ & $7.3215 \mathrm{e}-07$ & $4.6121 \mathrm{e}-07$ & $3.1576 \mathrm{e}-08$ \\
0.18 & $6.1861 \mathrm{e}-06$ & $1.4018 \mathrm{e}-06$ & $8.8705 \mathrm{e}-07$ & $6.1271 \mathrm{e}-08$ \\
0.27 & $8.6731 \mathrm{e}-06$ & $1.9601 \mathrm{e}-06$ & $1.2425 \mathrm{e}-06$ & $8.6100 \mathrm{e}-08$ \\
0.36 & $1.0471 \mathrm{e}-05$ & $2.3627 \mathrm{e}-06$ & $1.4992 \mathrm{e}-06$ & $1.0409 \mathrm{e}-07$ \\
0.45 & $1.1438 \mathrm{e}-05$ & $2.5777 \mathrm{e}-06$ & $1.6369 \mathrm{e}-06$ & $1.1382 \mathrm{e}-07$ \\
0.54 & $1.1496 \mathrm{e}-05$ & $2.5880 \mathrm{e}-06$ & $1.6445 \mathrm{e}-06$ & $1.1450 \mathrm{e}-07$ \\
0.63 & $1.0642 \mathrm{e}-05$ & $2.3928 \mathrm{e}-06$ & $1.5216 \mathrm{e}-06$ & $1.0610 \mathrm{e}-07$ \\
0.72 & $8.9419 \mathrm{e}-06$ & $2.0076 \mathrm{e}-06$ & $1.2779 \mathrm{e}-06$ & $8.9266 \mathrm{e}-08$ \\
0.81 & $6.5321 \mathrm{e}-06$ & $2.0077 \mathrm{e}-06$ & $9.3267 \mathrm{e}-07$ & $6.5346 \mathrm{e}-08$ \\
0.9 & $3.6036 \mathrm{e}-06$ & $8.0208 \mathrm{e}-07$ & $5.1338 \mathrm{e}-08$ & $3.6237 \mathrm{e}-08$ \\
1 & $1.2246 \mathrm{e}-16$ & $1.2246 \mathrm{e}-16$ & $1.2250 \mathrm{e}-16$ & $1.2246 \mathrm{e}-17$ \\
\hline
\end{tabular}

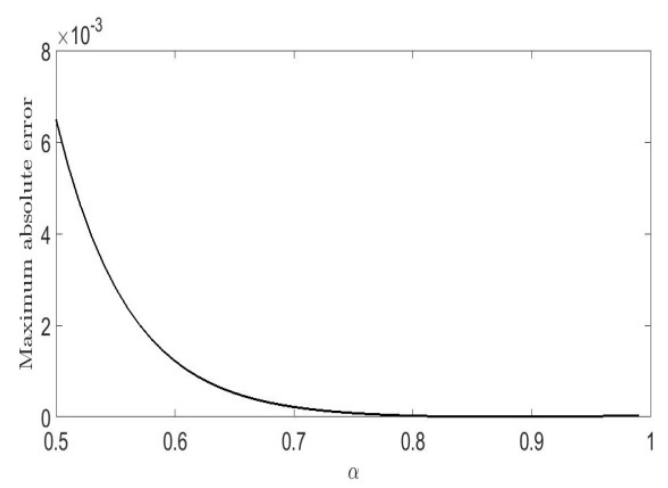

Figure 2. The maximum absolute error between our numerical solution and the analytical solution $u(x, t)=(t+1) \sin (\pi x)$ when $\alpha$ changes in the interval $[0.5,1)$. Number of grid points $M=200$.

\section{CONCLUSION}

In this paper we studied the numerical solution of a kind of an initial-boundary value problem of the time-fractional diffusion equation. The time-fraction order $\alpha$ of the diffusion equation has to be ranged in the interval $(0,1)$. The Galerkin finite element method is applied on the weak formulation of the problem. Time and space discretizations have been proceeded by using the linear B-spline (hat functions) as the basis functions. In our demonstrated numerical example, we found the absolute error is of order of $O\left(10^{-5}\right)$ for $\delta=0.9$ with $\delta_{x}=0.005$ and it decreased to order of $O\left(10^{-6}\right)$ for $\delta_{x}=0.0025$ by keeping time-step fixed. These confirm the reliability and efficiency of our proposed numerical method. Also we have found that this approach provides an approximate solution with the absolute error has order of $O\left(10^{-6}\right)$ for the values of $\alpha$ greater than 0.8 while this order increases as $\alpha$ decreases which then required a finer mesh to keep the absolute error on the order of $O\left(10^{-6}\right)$.

For the future study, one can work on increasing the length of the interval, shown in Figure 2, where the absolute error is insignificant, which is of great importance. This will widen the usability of the scheme for the problems govern by TFDE with $\alpha$ close to zero.

\section{REFERENCES}

Atangana A. (2015). Numerical analysis of time fractional three dimensional di_usion equation. Thermal Science, 19(suppl. 1):7-12.

Bellman R. (1997). Introduction to matrix analysis. Siam.

Esen, A., Uucar, Y., Yagmurlu, M., \& Tasbozan, O. (2014). Solving fractional diffusion and fractional diffusion-wave equations by Petrov-Galerkin finite element method. TWMS Journal of Applied and Engineering Mathematics, 4(2), 155-168.

Esen, A., Ucar, Y., Yagmurlu, N. and Tasbozan, O. (2013). A Galerkin finite element method to solve fractional diffusion and fractional diffusion-wave equations. Mathematical Modelling and Analysis, 18(2), pp.260-273.

http://dx.doi.org/10.3846/13926292.2013.783884

Esen, A., Tasbozan, O., Ucar, Y., \& Yagmurlu, N. M. (2015). A B-spline collocation method for solving fractional diffusion and fractional diffusion-wave equations. Tbilisi Mathematical Journal, 8(2), 181-193.

Tasbozan, O., Esen, A., Yagmurlu, N. M., \& Ucar, Y. (2013). A numerical solution to fractional diffusion equation for forcefree case. In Abstract and applied analysis, 2013. Hindawi. http://dx.doi.org/10.1155/2013/187383

Ferziger J. H. and Peric M. (2012). Computational methods for uid dynamics. SpringerScience \& Business Media.

Hanert E. and Piret C. (2012). Numerical solution of the space-time fractional diffusion equation:Alternatives to finite differences. In 5th IFAC Symposium on Fractional Differentiation and Its Applications-FDA2012.

Hanert E. (2010). A comparison of three eulerian numerical methods for fractional-order transport models. Environmental uid mechanics, 10(1-2):7-20.

Hilfer R. (2000). Applications of fractional calculus in physics. World Scientific.

Horn R. A. and Johnson C. R. (1991). Topics in matrix analysis. Cambridge University Presss, Cambridge, 37:39, 1991.

Kilbas A. A. et al (2006). Theory and applications of fractional differential equations, 204 (north-holland mathematics studies).

Lin, Z., Wang, D., Qi, D., \& Deng, L. (2020). A Petrov-Galerkin finite element-meshfree formulation for multi-dimensional fractional diffusion equations. Computational Mechanics, 66, 323-350. 
Ling L. and Yamamoto M. (2013). Numerical simulations for space $\{$ time fractional diffusion equations. International Journal of Computational Methods, 10(02):1341001.

Mainardi F. (1996). The fundamental solutions for the fractional diffusion-wave equation. Applied Mathematics Letters, 9(6):23-28.

Francesco Mainardi and Gianni Pagnini. The wright functions as solutions of the timefractional diffusion equation. Applied Mathematics and Computation, 141(1):5162,2003 .

Podlubny I. et al. (2009). Matrix approach to discrete fractional calculus II : Partial fractional differential equations.
Journal of Computational Physics, 228(8):3137-3153. ISSN 0021-9991. doi: 10.1016/j.jcp.2009.01.014. URL http://dx.doi.org/10.1016/j.jcp. 2009.01.014.

Samko S. G. et al. (1993). Fractional integrals and derivatives. Theory and Applications, Gordon and Breach, Yverdon.

Sun Z and Wu X (2006). A fully discrete difference scheme for a diffusion-wavesystem. Applied Numerical Mathematics, 56(2):193-209.

Tikhonov A. et al. (2013). Numerical methods for the solution of illposed problems. Springer Science \& Business Media. 